\title{
REVIEWS
}

JoAnna Kobierska-Brzoza $^{\text {A-D, }}$, Urszula KaCzmareK ${ }^{\text {A, B, E, F }}$

\section{Genetic Aspects of Dental Caries}

\section{Genetyczne aspekty próchnicy}

Department of Conservative and Pediatric Dentistry, Wroclaw Medical University, Wrocław, Poland

A - research concept and design; $\mathbf{B}$ - collection and/or assembly of data; C - data analysis and interpretation;

$\mathbf{D}$ - writing the article; $\mathbf{E}$ - critical revision of the article; $\mathbf{F}$ - final approval of article

\begin{abstract}
Dental caries still remain one of the most prevalent infectious diseases, affecting populations and individuals unequivocally. It is observed that people with similar behavioral risk present different caries rates. Susceptibility to caries results from both the host and environmental factors that complement each other and may result in increased incidence of caries or greater resistance to it. Numerous studies of twins, families and animal models initiated in the early decades of the previous century provided strong evidence for the genetic component of dental caries development. The succeeding research, identifying specific genes and genetic markers and confirming the genetic correlation of dental caries, explained the reasons behind the greater susceptibility to caries in some diseases like Epidermolysis bullosa. Many inherited determinants are well established. The most recognizable are the structure of the hard dental tissue, tooth morphology including depth of occlusal fissures and surface characteristic of the enamel, time of eruption, alignment of the teeth in dental arches, flow rate of the saliva and its constituents, immunologic response to cariogenic microorganisms in oral cavity. An important role is also played by inherited thresholds for tastes and smells, which affect taste preferences and thus dietary habits and activity of specific enzymes participating in regulation processes on various stages of development and subsequent physiologic functioning of the organism. The knowledge of genetic basis of caries will enable us to identify high-risk groups and provide them with targeted screening, preventive measures and interventional strategies (Dent. Med. Probl. 2016, 53, 3, 413-418).
\end{abstract}

Key words: genetics, dental caries, genes.

Słowa kluczowe: genetyka, próchnica, geny.

Dental caries is a commonly distributed disease in the world. Conventionally, it is considered to be an infectious, transmissible and multifactorial disease caused by well-recognized causative factors, such as cariogenic bacteria, fermentable carbohydrates, which interact with the host for the period of time needed to initiate the process. However, such model oversimplifies the complexity of the disease, as the examples of some people do not confirm this definition (a patient who consumes a lot of sugar does not always suffer from caries and other patients who consume little sugar present high risk for caries). Therefore, various behavioral and socioeconomic factors must be taken into consideration when assessing the likelihood of caries lesion development in an individual and the population. Currently, the etiology of caries disease is thought to be a complex interplay of the oral environment and genetic factors. Observations show that development of caries depends on the complex interactions between the individual genetic profile and superimposing environmental factors. Heritability of caries was the subject of interest from the beginning of the past century and was investigated from various angles, and the underlying genetic role in caries susceptibility has been suggested. One of the first studies on the genetic contribution focused on caries prevalence among twins, because this model of study permitted researchers to distin- 
guish between the environmental and genetic impact on caries development. Those previous studies compared the prevalence and intensity of carious process in dizygotic and monozygotic twins and showed great similarity of the carious process in monozygotic twins as well as considerable diversity among dizygotic twins of various sex $[1,2]$. In the early 1930s, Goldberg even identified the occurrence of carious lesions in corresponding teeth among monozygotic twins. These observations led him to conclude that there is a significance of genetic factors in the prevalence of caries [3]. Nevertheless, in those years the role of genetic influence was limited to the hereditary impact on teeth morphology, their alignment in the arches, the shape of the dental arch, the sequence and timing of eruption as well as salivary composition and flow rate. Subsequent studies confirmed an even greater similarity in caries history of monozygotic twins compared to dizygotic ones [4-6]. The greatest differences concerned the lesions on the smooth surfaces. One of the shortcomings of these studies was the fact that they involved children and young people who usually lived in the same family and thus shared the same oral environmental factors with respect to diet and oral hygiene practices. Hence, consecutive studies included older participating twins from various regions and habitats $[6,7]$. These analyses also disclosed a similarity in the prevalence and localizations of carious lesion both in monozygotic as in dizygotic twins, although that similarity could mean increased or decreased susceptibility. In search for genetic backgrounds of caries, researchers turned to animal models, which were also analyzed after crossing the strains susceptible to caries with the resistant ones. These studies allowed us to formulate a hypothesis suggesting a significant role of certain specific loci in chromosomes 1,2,7,8 in regard to a general proneness to caries [8-11]. For many years, effort was made to establish the existence of specific genes directly responsible for high or low caries susceptibility in a particular individual.

Among several studies, probably the most recognizable is the classic Vipeholm study, in which it was observed that $20 \%$ of participants exposed to a diet rich in high carbohydrates snacks did not develop any new carious lesions during the analyzed period of time, whereas others demonstrated dynamic progression of caries process [12]. This indicated that this subpopulation had better resistance.

Evidence of genetic contribution to caries incidence was also provided by Vieira [13], who assessed the genotype DMF in 46 selected families from Philippines, and on the basis of this data typ- ified three regions related to low caries susceptibility (5q13,3, 14q11,2 and Xq27,1) and two regions characterized by high incidence of caries (13q31,1 and 14q24,3).

Well-recognized genetic determinants of proneness to caries include (Fig. 1) the structure of hard dental tissues, particularly the degree of their mineralization, immunologic response or rather variations of immunologic response to cariogenic bacteria in oral cavity, composition and flow rate of saliva, metabolism and the intake of carbohydrates in the diet [5].

\section{Impact \\ on Enamel Formation}

Hard dental tissues are submitted to modulating effects related to heredity on the many stages of their development. One of the initial ones is amelogenesis. Genetically controlled production of enamel matrix proteins plays a significant role during the formation of the enamel. These proteins are commonly divided into amelogenins and non-amelogenins. The latter include enamelins, ameloblastins and tuftelins. Similarly, like other proteins in the organism, they are genetically coded and demonstrate some variation.

The biggest part, about $90 \%$ of the enamel matrix proteins, constitute amelogenins. Their genes are localized on the $p$ arm of chromosome $\mathrm{X}$ (AMELX) in loci Xp22, 31-p22, 1. The males have the equivalent of this gene on the chromosome $Y$ (AMELY). It has been proved that deletion or mutation of the gene AMELX is responsible for the developmental disorder known as imperfect amelogenesis related to chromosome X. Mutations of the genes lead finally to a deficiency of amelogenins, disturbing the formation of the enamel matrix and, in consequence, contributing to lower resistance to caries of defected enamel [6]. It has been also confirmed that the presence of allele $\mathrm{C}$ of amelogenin gene correlates with higher scores of DMF. The gene protective to caries incidence in locus q 27 on chromosome $\mathrm{X}$ was identified [14, 15].

Ameloblastins may also influence the vulnerability of enamel to caries. The highest concentration of these proteins occurs during differentiation of enamel organ into ameloblasts and they are localized mainly in Tomes processes of ameloblasts. Another protein participating in the formation of the enamel matrix and the early stages of mineralization are tuftelins. A significant amount of these proteins are secreted near the dentino-enamel junction. Excessive expression of tuftelins' gene, resulting in increased produc- 


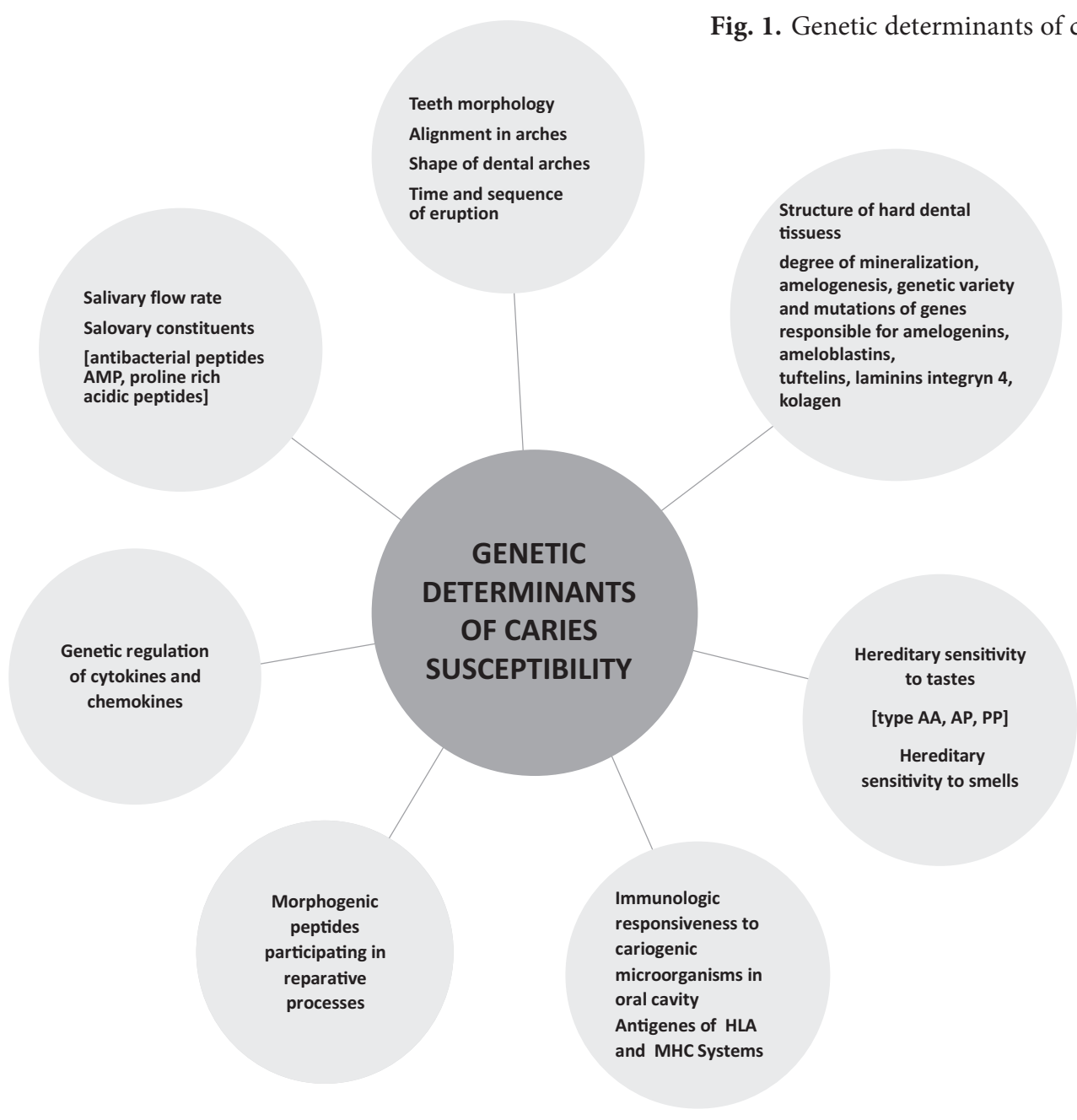

tion of these proteins, leads to disturbances in the structure of enamel prisms and crystalic structure of the enamel. Mutations of the genes coding the sequence of tuftelins may as well indirectly influence susceptibility to caries through interactions with other proteins $[14,15]$. Moreover, the studies showed a relation between allele T of tuftelins' gene and increased scores of the DMF index and between the genetic variety of tuftelins and amelogenins and higher prevalence of caries [14].

Genes of HLA complex also play a significant role in the formation of the enamel. The presence of HLA-DR3 was confirmed to associate more frequently with enamel defects and greater susceptibility to dental caries, whereas the presence of gene HLA DR5 was related with a reduced occurrence of enamel abnormalities. Epidermolysis bulosa (EB) is probably the best-documented example of direct genetic influence on anomalies in dental hard tissues, which lead to lower resistance to caries. The alterations in the structure of the enamel, involving greater porosity and compromised mineralization, result in greater carries incidence among these patients [16]. These defects are the result of a mutation of one of 3 genes re- sponsible for the synthesis of laminin 5, 4 integin and type XVII collagen. Disturbances in crystal formation and an increased amount of serum albumins lead to greater porosity of defected enamel, which increases the surface exposed to demineralization, while simultaneously lowering the enamel's ability to remineralize. Epidermolisis bulosa proves that mutations of the genes may induce direct alterations in the tooth structure, which influence their resistance to caries [17].

\section{Impact on Host Response to Oral Bacteria}

Genetic influence of the immune response involves great diversity of modifications, which determine enormous individual variety in the degree of colonization of the oral cavity by cariogenic microorganism, especially by Streptococcus mutans. This is in particularly visible among the patients with different congenital or acquired immunodeficiencies.

Analyses of the association between the susceptibility to dental caries measured with the val- 
ues of the DMFS index and HLA complex (Human Leukocyte Antigen) and MHC complex (Major Histocompatibility Complex) showed unequivocal results. Lehner et al. [18] observed a significant relationship between the antigen HLA DRW6, the DMF score and the lack of such correlation with antigen HLA DR4. Other authors noticed the genes from MHC complex, especially DR4 and DR3 in persons with a high level of $S$. mutans in the oral cavity [19]. De Vries et al. [20], in turn, discovered no relation between caries incidence and the type of HLA DR. The study carried out by Senpuku et al. [21] and Acton [22] proved the correlation of certain types of HLA DR with surface antigens of S. mutans and the colonization of these cariogenic pathogens on teeth. Alterations within MHC can modify the host immune response to $S$. mutans. These associations were confirmed in patients with celiac disease by Aine [23] and Aiguirre et al. [24]. They discovered the affiliation between HLADR3 and the presence of defects in the enamel predisposing it to the development of caries lesions among these patients. The mentioned studies provided evidence of an association between the genes of HLA complex and disturbances in the development of enamel and its greater vulnerability to caries. It was also observed that subjects with antigen HLADRB $1{ }^{\star} 04$ demonstrated a ten times greater susceptibility to early childhood caries (ECC) than those without this antigen. The role of beta defensins (DEFB), which allele correlated with high or lowered values of DMF remains beyond a doubt important, although is certainly unclear [5].

\section{Impact on Intake and Metabolism of Carbohydrates}

There are not many reports about the influence of hereditary metabolic disorders on the incidence and progress of caries. The most recognizable disorder is congenital intolerance of fructose caused by the deficiency of fructose-1 phosphatase aldolase. Affected patients experience serious somatic symptoms after sugar intake, including lifethreatening conditions. Low susceptibility to caries observed among these persons is rather related to the implementation of restricted diets than caused by genetic mechanisms [25].

However, consumption of carbohydrates in a diet beyond its obvious behavioral aspects is certainly related to the perception of taste and smell of food. Taste preferences which affect dietetic habits are related to individual sensitivity, which is in- herited $[26,27]$. Congenital thresholds play an important role for specific tastes. Mennella et al. [28] analyzed genes responsible for the receptors of bitter taste, and based on analyzed genotypes they distinguished three possible types. Type AA were individuals who did not taste standard substance of phenylthiocarbamide and had two recessive alleles. They were not sensitive to bitter taste. Type PP included people very sensitive to bitter taste of phenylthiocarbamide and have two dominant alleles. The last intermediate type AP involved individuals with one allele from each previous group. Sensitivity to bitter taste is inherited. Those with high sensitivity and low taste threshold recognize the tastes in considerably lower concentrations, whereas people with a higher taste threshold require greater concentrations of sweet or bitter substance in food to be able to perceive its taste. For these reasons their consumption of sweets can be relatively greater, which potentially constitutes higher risk of caries. The higher scores of DMF among the nontasters were observed by Lin [29]. Knowledge of sensitivity to tastes can be useful in evaluating individual risk to caries.

Similarly, variety of genes responsible for smell receptors may influence the perception of smell, herein the smell of food, which in turn may explain the predilections to specific meals resulting in more or less cariogenic diet.

\section{Impact \\ on Salivary Secretion}

Secretion of the saliva and its composition are other factors whose genetic origin has the potential to modify individual susceptibility to dental caries. Salivary flow rate is lower in females than in males. This fact increases the risk of caries in females, because they benefit less from the protective effects of saliva, which include dissolution, clearance, buffering, remineralization and antibacterial activity. The role of optimal physiologic composition of the saliva necessary to meet all these requirements cannot be overestimated.

Salivary proteins fulfill various functions in the complex interactions between microorganisms of the oral cavity and the host. All of them are determined genetically.

Acidic proline-rich proteins (PRPs) play an important role in attaching the cariogenic microorganism to the surfaces of the teeth. These polymorphic proteins attach themselves to the surface of the tooth with the one of its domains, exposing another terminal domain for binding with microorganisms, which initiates the formation of dental plaque. All these proteins are coded by blocks 
of genes localized on the short arm of chromosome 12, at two loci, PRH1 and PRH2 and their polimorphism is provided by three alleles in each of the listed loci. Two specific phenotypes of PRP are associated with a higher incidence of caries in adults, whereas the other two alleles are related to lower morbidity [30].

Antimicrobial Peptides AMP 1 are the first line in the salivary defense system. Their concentration in the saliva demonstrates great individual variety and is determined genetically. On the basis of their structure and composition, three following groups were distinguished: alpha helical peptides without cysteine (cathelicidins), peptides containing three disulfide bonds (alpha and beta defensins) and peptides with high contents of certain specific aminoacids [31]. The high concentration of defensins HNP1-3 was observed in children free from caries, so the level of genetically determined AMP could serve as one of the predicators of caries risk in children [31].

\section{Impact on Immune Response}

Identification of the genes responsible for caries susceptibility of adults was the goal of the pioneer Genome Wide Association Study (GWAS) conducted by Wang et al. [32]. They assessed in meta analyses the caries incidence and genetic markers (polimorphisms of single nucleotydes) typified within the whole genome and related to caries susceptibility. They confirmed the relation of gene rs 635808 with chromosome 6 in region RPS6KA2. This gene is responsible for the enzyme which participates in the regulation of cytokines and chemokines that are present in various pathologic processes in oral cavity, among them, also dental caries. The obtained results also revealed the important role played by gene PTK2B in the pathologic processes. Another case concerned gene BMP4 coding morphogenetic proteins, which participate in the reparative processes of dentine-pulpal complex after carious destruction. Bmp4 initiates and regulates the reparative processes.

The authors also observed a suggestive relation between the gene described as RHOU, which is localized on chromosome 1, with the regulation of tooth morphology during its developmental stage. The important role in tooth development is also attributed to the gene ADMTS3 localized on the chromosome 4; especially the relation to polymorphism of nucleotides in the region ADMTS3 and ISL1 was confirmed [29]. Despite wide scale of the GWAS Project, which included tree meta-analyses of all collected data, the authors did not reveal specific genes directly responsible for the development of caries, but they confirmed the activity and role of certain specific genes in the regulative processes.

The results of the mentioned papers can suggest a strong genetic contribution to the susceptibility to caries in multifactorial etiology of caries, although the impact of environmental and behavioral factors makes the interpretation of the genetic affiliations more difficult. Further investigation of the human genome and improvement of methodology of such studies will certainly permit us to better recognize the genetic background and to formulate more efficient and customized strategies of diagnosis, risk assessment and treatment.

\section{References}

[1] Shuler Charles F.: Risks for susceptibility to dental caries. J. Dent. Educ. 2001, 65, 1038-1045.

[2] Bretz W.A., Corby P., Schork N., Hart T.: Evidence of a contribution of genetic factors to dental caries risk. J. Evid. Based Dent. Pract. 2003, 3, 185-189.

[3] Fairpo C.G.: Total caries experience in monozygotic and ike-sexed dizygotic twins of Caucasoid origin aged 5 to 15 years. Arch. Oral Biol. 1979, 24, 491-494.

[4] Bordoni N., Dono R., Manfredi C., Allegrotti I.: Prevalence of dental caries in twins. J. Dent. Child. 1973, 40, 440-443.

[5] Bretz W.A., Corby P., Hart T., Costa S., Coelho M.Q., Weyant R.J., Robinson M., Schork N.: Dental caries and microbial acid production in twins. Caries Res. 2005, 39, 168-172.

[6] Conry J.P., Messer L.B., BoraAs J.C., Aeppli D.P., Bouchard T.J.: Dental caries and treatment characteristics in human twins reared apart. Arch. Oral Biol. 1993, 38, 937-943.

[7] Boraas J.C., Messer L.B., Till M.J.: A genetic contribution to dental caries, occlusion and morphology as demonstrated by twins reared apart. J. Dent. Res. 1988, 67, 1150-1155.

[8] Opal S., Garg S., Jain J., Walia I.: Genetic factors affecting dental caries risk. Aust. Dent. J. 2015, 1, 2-11.

[9] Suzuki N., Kurihara Y., Kurihara Y.: Dental caries susceptibility in mice is closely linked to the H-2 region on chromosome 17. Caries Res. 1998, 32, 262-265.

[10] Uematsu T., Nariyama M., Shimizu K., Maeda T.: Mapping of affected gene(s) to dental caries susceptibility on mouse chromosome 2. Pediatr. Dent. J. 2003, 13, 75-81.

[11] Nariyama M., Shimizu K., Uematsu T., Maeda T.: Identification of chromosomes associated with dental caries susceptibility using quantitative trait locus analysis in mice. Caries Res. 2004, 38, 79-84. 
[12] Krasse B.: The Vipelolm Dental Caries Study: Recollections and reflections 50 years later. J. Dent. Res. 2001, 80, 1785-1788.

[13] Vieira A.R.: Genetics and caries - prospects. Braz. Oral Res. 2012, 26, 7-9.

[14] Renuka P., Pushpanjali K., Sangeetha R.: Review on “influence of host genes on dental caries." J. Dent. Med. Sci. 2013, 4, 86-92.

[15] Patir A., Seymen F., Yildirim M., Deeley K., Cooper M.E., Marazita M.L.: Enamel formation genes are associated with high caries experience in Turkish children. Caries Res. 2008, 42, 394-400.

[16] Kirkham J., Robinson C., Strafford S.M., Shore RC., Bonass W.A., Brookes S.J., Wright J.T.: The chemical composition of tooth enamel in junctional epidermolysis bullosa. Arch. Oral Biol. 2000, 45, 377-386.

[17] Timothy Wright J.: Oral manifestation in the Epidermolysis Bullosa spectrum. Dermatol. Clin. 2010, 1, $159-164$.

[18] Lehner T., LAmb J.R., Welsh K.L., BAtchelor R.J.: Association between HLA-DR antigen and helper cell activity in the control of dental caries. Nature, 1981, 292, 770-772.

[19] Bagherian A., Nematollahi H., Afshari J.T., Moheghi N.: Comparison of allele frequency for HLA-DR and HLA-DQ between patients with ECC and caries-free children. J. Indian Soc. Pedod. Prevent. Dent. 2008, 26, 18-21.

[20] De Vries R.R., Zeylemaker P., van Palenstein-Helderman W.H., Huis Veld J.H.: Lack of association between HLADR antigens and dental caries. Tissue Antig. 1985, 25, 173-174.

[21] Senpuku H., Yanagi K., Nisizawa T.: Identification of Streptococcus mutans PAc peptide motif binding with human MHC class II molecules (DRB1-0802, 1101, 1401 and 1405). Immunol. 1998, 95, 322-330.

[22] Aстоn R.T.: Association of MHC genes with levels of caries inducing organisms and caries severity in African American women. Human Immunol. 1999, 60, 984-989.

[23] Aine L.: Coeliac-type permanent-tooth enamel defects. Annal. Med. 1996, 28, 9-12.

[24] Aguirre J.M., Rodriquez R., Oribe D., Vitoria J.C.: Dental enamel defects in celiac patients. Oral Surg. 1997, $84,646-650$.

[25] Sofaer J.A.: Host genes and dental caries. Br. Dent. J. 1993, 175, 403-409.

[26] Rupesh S., NAyak U.A.: Genetic sensitivity to the bitter taste of 6-n propylthiouracil: A new risk determinant for dental caries in children. J. Indian Soc. Pedod. Prev Dent. 2006, 4, 63-68.

[27] Bhasin M.K.: Genetics of castes and tribes of India: Taste sensitivity. Int. J. Hum. Genet. 2006, 6, 145-151.

[28] Mennella J.A., Pepino M.Y., Reed D.R.: Genetic and environmental determinants of bitter perception and sweet preferences. Pediatr. 2005, 1115, 216-222.

[29] Lin B.P.: Caries experience in children with various genetic sensitivity levels to the bitter taste of 6-n-propylinthiouracil (PROP). Pediatr. Dent. 2003, 25, 37-42.

[30] Zakhary G.M., Clark R.M., Bidichandani S.I., Owen W.L., Slayton R.L., Levine M.: Acidic proline-rich prorotein $\mathrm{Db}$ and caries in young children. J. Dent. Res. 2007, 86, 1176-1180.

[31] Renchuan T., Jurevic R., Coulton K., Marjorie T.: Salivary antimicrobial peptide expression and dental caries experience in children. Antimicrob. Agents Chemother. 2005, 49, 3883-3888.

[32] Wang X., Shaffer J.R., Zeng Z., Begum F.,Vieira A.R., Noel J., AnjomshoaA I., Cuenco T.K., Lee M.K., Beck J., Boerwinkle E., Cornelis M.C., Frank E., Hu B., Crosslin D.R., Laurie C.C., Nelson S.C., Doheny K.F., Pugh E., Polk E., Weyant R., Crout R., McNeil D., Weeks D.E., Feingold E., Marazita M.L.: Genome wide association scan of dental caries in the permanent dentition. BMC Oral Health, 2012, 12, 57.

\section{Address for correspondence:}

Joanna Kobierska-Brzoza

Department of Conservative and Pediatric Dentistry

Wroclaw Medical University

Krakowska 26

50-425 Wrocław

Poland

E-mail: joanna.kobierska-brzoza@umed.wroc.pl

Conflict of Interest: None declared

Received: 12.03 .2016

Revised: 4.04.2016

Accepted: 29.04.2016 